\title{
Uma Nota sobre Erros de Previsão da Inflação de Curto-Prazo*
}

\author{
Emanuel Kohlscheen ${ }^{\dagger}$
}

\author{
Conteúdo: 1. Introdução; 2. Testando o Viés e a Racionalidade das Previsões; 3. Histerese \\ das Expectativas; 4. Previsões Ajustadas; 5. Conclusões. \\ Palavras-chave: Previsão; Expectativas racionais; Expectativas Adaptativas; Rigidez de \\ informação; Inflação. \\ Códigos JEL: E31; E37
}

Apesar de testes não rejeitarem as hipóteses de ausência de viés e racionalidade fraca nas previsões de inflação, existe um padrão de autocorrelação nos erros das previsões. Aumentos (diminuições) na taxa de inflação estão sistematicamente associados a sub-estimações (sobreestimações) da inflação no período seguinte. Os testes sugerem que os modelos em que observações passadas da inflação têm maior peso na composição de expectativas correntes (como modelos de expectativas adaptativas ou de rigidez de informação), têm melhor poder explicativo. Modelos que procurem explicar a formação de expectativas devem ser capazes de explicar estes fatos estilizados bem como a histerese nas projeções.

This note shows that the unbiasedness and the weak rationality hypotheses are not rejected for inflation forecasts surveyed by the Central Bank. However, a clear pattern of auto-correlation of forecast errors is found. Furthermore, increases (decreases) in inflation are systematically associated with underestimations (overestimations) of inflation in the following month. This suggests that models in which past realizations of inflation have greater weight in the formation of expectations are more accurate than the assumption of rational expectations. Models aimed at explaining how expectations are formed should be able to explain these stylized facts as well as the hysteresis of forecasts.

\footnotetext{
${ }^{*}$ Agradeço a Adriana Soares Sales, Wagner Piazza Gaglianone e a um parecerista anônimo por seus comentários e sugestões. As opiniões expressas neste trabalho são exclusivamente do autor e não refletem, necessariamente, a visão do Banco Central do Brasil.

†Departamento de Estudos e Pesquisas, Banco Central do Brasil. Setor Bancário Sul, Quadra 3, Bloco B, 70074-900 Brasília-DF, Brasil. E-mail: emanuel.kohlscheen@bcb.gov.br
} 


\section{INTRODUÇÃO}

A teoria de expectativas racionais dita que, ao receber nova informação que afete o comportamento futuro de uma variável econômica, o agente econômico deve revisar de imediato todas as suas expectativas quanto à variação futura desta variável a fim de incorporar esta nova informação. Deste modo, quando não há barreiras à disseminação da informação, uma revisão das expectativas de inflação para cima deveria necessariamente estar associada à chegada de informação (negativa) nova que ainda era desconhecida pelos agentes econômicos quando a estimativa anterior foi feita.

A observação casual nos permite inferir que o modelo de expectativas racionais com informação completa tem dificuldades de explicar certos comportamentos das expectativas de inflação divulgadas pelo Banco Central. Considere, por exemplo, o fato de que o Relatório de Mercado do último dia 21 de maio (de 2010) registrou o décimo oitavo aumento semanal consecutivo da expectativa mediana de inflação para 2010. Em um cenário de inflação estável, onde existisse igual probabilidade de notícias positivas e negativas, a probabilidade de uma seqüência destas ocorrer em um período de 18 semanas qualquer é de apenas $0,00038 \%$ (isto é, aproximadamente uma vez a cada 5.000 anos). ${ }^{1}$

Configuram-se então duas alternativas: ou o modelo de expectativas racionais com informação completa é inadequado para descrever o contexto em questão, ou o período atual constitui um ciclo de aceleração da inflação sem precedentes. Como veremos que não há evidências de mudança no regime da inflação no período analisado, este estudo examina a formação de expectativas por parte dos agentes econômicos. Como mostraremos, embora as hipóteses de ausência de viés e racionalidade fraca das expectativas não são rejeitadas, encontramos um claro padrão de auto-correlação nos erros de previsão que são dificeis de serem explicados num contexto de expectativas racionais ou mesmo de rigidez de informação à la Mankiw e Reis.

\section{TESTANDO O VIÉS E A RACIONALIDADE DAS PREVISÕES}

Começamos testando a inexistência de viés e a racionalidade das previsões que compõem a amostra utilizada para a divulgação semanal das estatísticas no boletim Focus, do Banco Central do Brasil. A fim de se testar a hipótese de que os agentes econômicos que participam da pesquisa não cometem erros sistemáticos em suas projeções consideram-se aqui as projeções de variação mensal do Índice Nacional de Preços ao Consumidor Amplo (IPCA) feitas um mês antes da data de divulgação do dado. Ressalte-se que o survey do Banco Central utilizado para tal teste apresenta algumas vantagens consideráveis sobre equivalentes encontrados em outros países. Por exemplo, o tradicional Michigan survey nos Estados Unidos coleta expectativas de inflação sem sequer especificar o índice de inflação ao participante.

\subsection{Testes para a Amostra Completa}

Como de praxe, as equações estimadas para se testar o viés e a racionalidade das expectativas relatadas no Focus foram, respectivamente,

$$
\pi_{t}-E\left[\pi_{t} \mid I_{t-1}\right]=\alpha_{0}+\eta_{t}
$$

e

$$
\pi_{t}=\beta_{0}+\beta_{1} E\left[\pi_{t} \mid I_{t-1}\right]+\varepsilon_{t},
$$

\footnotetext{
${ }^{1}$ Alternativamente, a probabilidade de que uma série histórica de 100 observações mensais - como a que utilizamos neste estudo - contenha uma seqüência de 18 altas semanais consecutivas é de 1 em 612. Note que, para chegar a estes números, ignoramos a possibilidade de que a expectativa de inflação não varie. Se levássemos esta possibilidade em conta, as probabilidades seriam ainda menores.
} 
onde $\pi_{t}$ corresponde à inflação mensal, $I_{t-1}$ representa o universo de informações disponíveis no instante em que a previsão é feita (isto é, em $t-1$ ) e $E$ representa o operador que diz respeito às expectativas. A estimação da primeira equação visa verificar a ausência de um viés sistemático e significativo nas projeções, enquanto na segunda equação a forma fraca da racionalidade é verificada ao se testar a hipótese nula de que $\beta_{0}=0$ e $\beta_{1}=1$. As estimativas foram feitas para o período de janeiro de $2002 \mathrm{a}$ abril de 2010 (ou seja, empregando 100 observações mensais). ${ }^{2}$

Tabela 1: Testes de Viés e de Racionalidade das Expectativas

\begin{tabular}{|c|c|c|c|c|}
\hline \multicolumn{5}{|c|}{$\alpha_{0}$} \\
\hline \multirow[t]{3}{*}{ Mediana } & 0,070 & & & \\
\hline & $(0,050)$ & & & \\
\hline & $\beta_{0}$ & $\beta_{1}$ & $X^{2}$ & valorp \\
\hline \multirow[t]{2}{*}{ Mediana } & $-0,134$ & $1,435^{* * *}$ & 2,80 & 0,247 \\
\hline & $(0,107)$ & $(0,290)$ & & \\
\hline \multicolumn{5}{|c|}{ Nota: Os desvios-padrão foram corrigidos utilizando-se } \\
\hline a metodolo & de Newe & West $(1987$ & & \\
\hline
\end{tabular}

A Tabela 1 mostra que a expectativa mediana do IPCA mensal tipicamente sub-estima a inflação mensal em 7 pontos-base. Este viés, no entanto, não é estatisticamente significativo quando utilizamos desvios-padrão que são robustos à heteroscedasticidade e auto-correlação, conforme sugerido por Newey e West (1987). Em função da grande magnitude dos desvios-padrão corrigidos, o teste de Wald não nos permite rejeitar a hipótese da racionalidade fraca da mediana das expectativas: a estatística $\chi^{2}$ é igual a 2,80, estando associada a um valor $p$ de 0,247 .

\subsection{Persistência dos Erros de Previsão}

A não-rejeição da versão fraca da racionalidade pelo teste de viés, no entanto, deve ser considerada com cautela. A Tabela 2 a seguir mostra que os erros de previsão apresentam auto-correlação bastante forte e persistente - o que parece inconsistente com a hipótese de expectativas racionais com informação completa. A coluna $\ell(3)$ mostra a estatística obtida do teste de Cumby e Huizinga (1992), que tem distribuição $\chi^{2}(3)$. As estatísticas claramente indicam a rejeição da hipótese nula de que a primeira até a terceira auto-correlação dos erros de previsão são zero. Poder-se-ia contra-argumentar que um dos motivos pelo qual os agentes parecem estar cometendo erros sistemáticos e previsíveis é que o período de teste inclui a crise de confiança de 2002. A metade inferior da tabela, no entanto, descarta esta hipótese, mostrando que a auto-correlação continua presente mesmo quando a primeira metade da série histórica é descartada.

A sugestão aqui é de que as expectativas não são eficientes, uma vez que a informação relevante sobre os erros de estimativas anteriores aparentemente é ignorada. Em outras palavras, a versão forte da racionalidade é claramente rejeitada. De fato, as correlações positivas na tabela indicam que aumentos (diminuições) na taxa de inflação estão sistematicamente associados a sub-estimações (sobre-) da

\footnotetext{
${ }^{2} \mathrm{O}$ período analisado neste estudo inicia-se em 2002 porque a série histórica das projeções das instituições Top 5 (ranking de curto prazo) se inicia somente no final de 2001.
} 
Tabela 2: Características dos Erros de Previsão - Mediana da Amostra

\begin{tabular}{lcccc}
\hline \multicolumn{5}{l}{ correlação dos erros de previsão com $x_{t}$ na defasagem $s$} \\
\hline \multicolumn{4}{l}{ desde janeiro de 2002 (100 observaçoes) } \\
\hline$x_{t}$ & $s=1$ & $s=2$ & $s=3$ & $\ell(3)$ \\
\cline { 2 - 5 }$\pi_{t}-E\left[\pi_{t} \mid I_{t-1}\right]$ & 0,576 & 0,308 & 0,197 & $33,30^{* * *}$ \\
$\Delta \pi_{t}$ & 0,300 & 0,134 & 0,057 & \\
\hline \multicolumn{4}{l}{ desde março de 2006 (50 últimas observações) } \\
\hline$x_{t}$ & $s=1$ & $s=2$ & $s=3$ & $\ell(3)$ \\
\cline { 2 - 5 }$\pi_{t}-E\left[\pi_{t} \mid I_{t-1}\right]$ & 0,403 & 0,182 & 0,018 & $8,77^{* *}$ \\
$\Delta \pi_{t}$ & 0,172 & 0,108 & $-0,018$ & \\
\hline
\end{tabular}

Nota: A estatística $\ell(3)$ é o resultado do teste em que a hipótese

nula sustenta que a primeira até a terceira auto-correlação dos

erros de previsão é zero.

*** $\mathrm{e}^{* *}$ indicam significância estatística no nível de confiança de

$1 \%$ e $5 \%$, respectivamente.

inflação no período seguinte. Este padrão pode ser verificado também no gráfico a seguir, que mostra a média móvel da surpresa inflacionária, $\pi_{t}-E\left[\pi_{t} \mid I_{t-1}\right]$, bem como a aceleração média da inflação definida como a diferença entre a inflação média nos 3 últimos meses em relação aos 3 meses imediatamente anteriores.

Como se vê, episódios de aceleração de inflação tendem a estar associados a surpresas inflacionárias.

Vale lembrar aqui que Evans e Wachtel (1993) levantaram a possibilidade de que erros de previsão podem ser persistentes mesmo sob expectativas racionais desde que agentes antecipem uma possível mudança no regime de inflação. Esta explicação para a forte auto-correlação nos erros de previsão, no entanto, não nos parece ser satisfatória para o caso em questão, porque - diferentemente do que seria predito pelo modelo de Evans e Wachtel - não encontramos evidência de um viés significativo nas previsões no período recente.

Para as 50 observações mais recentes (isto é, março de 2006 a abril de 2010), a hipótese nula de que $\alpha_{0}=0$ na equação (1) claramente não pode ser rejeitada (valor $p$ de 0,45 ). Além disso, os gráficos no Anexo do trabalho para discussão que precedeu este artigo (Kohlscheen, 2010) mostram que os testes de estabilidade dos resíduos de Brown et alii (1975) também rejeitam a hipótese de instabilidade dos coeficientes no período recente.

\subsection{Testes para as Instituições Top 5}

Uma vez que a análise anterior apresentou evidências que contrariam a hipótese da racionalidade das expectativas nas projeções de curto prazo, poderíamos testar se a hipótese da racionalidade é válida ao menos para um subgrupo da amostra. Vale aqui lembrar que a teoria da "rigidez de informação" (RI) proposta em Mankiw e Reis (2002) e em Mankiw et alii (2004) sugere que um dos motivos pelo qual expectativas podem não parecer racionais é que apenas uma fração dos agentes econômicos atualiza suas projeções em um dado momento. Deste modo, apenas as expectativas dos agentes que atualizam suas projeções num dado instante se conformariam à teoria de expectativas racionais com informação completa. A fim de testar se a teoria de RI apresenta maior poder explicativo, repetimos os testes acima 
Figura 1: Surpresas Inflacionárias e Aceleração da Inflação

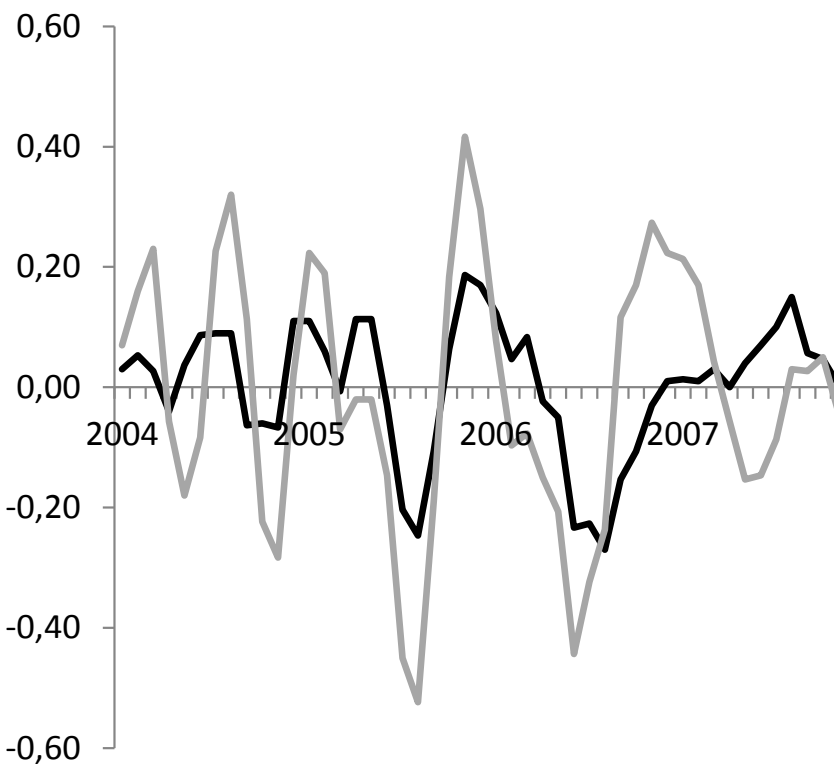

- MM 3 meses da surpresa inflacionária

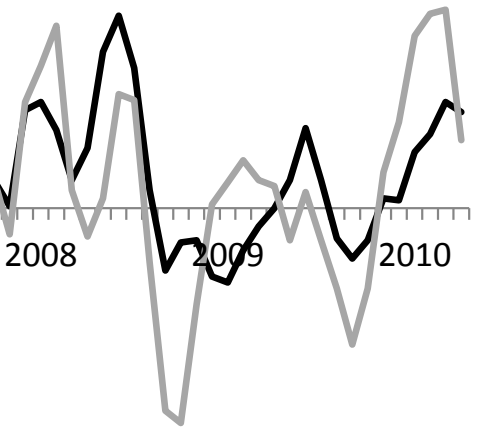

Aceleração trimestral

utilizando apenas as projeções das instituições classificadas como Top $5 .{ }^{3}$ Nossa expectativa é de que, se os melhores economistas-chefe atualizam suas informações e projeções de forma mais freqüente do que os economistas das demais instituições e, se a teoria de Mankiw e Reis estiver correta, observaremos maior consistência das projeções das instituições Top 5 com a teoria de expectativas racionais. As tabelas 3 e 4 mostram os resultados destes testes.

Tabela 3: Testes de Viés e Racionalidade das Expectativas - Instituições Top 5

\begin{tabular}{ccccc}
\hline \multicolumn{5}{c}{$\alpha_{0}$} \\
& & & \\
& 0,048 & & & \\
$(0,043)$ & & & \\
\hline Mediana & $\beta_{0}$ & $\beta_{1}$ & $X^{2}$ & valorp \\
\hline & $-0,092$ & $1,286^{* * *}$ & 2,51 & 0,285 \\
& $(0,070)$ & $(0,183)$ & & \\
\hline
\end{tabular}

Nota: Os desvios-padrão foram corrigidos utilizando-se

a metodologia de Newey e West (1987).

*** indica significância estatística ao nível de confiança de $1 \%$.

\footnotetext{
${ }^{3} \mathrm{O}$ ranking utilizado aqui foi o de curto prazo mensal.
} 
Como pode-se ver, a restrição da amostra às instituições Top 5 de fato aproxima os parâmetros estimados dos valores que seriam os condizentes com a racionalidade das expectativas (isto é $\alpha_{0}=0$, $\beta_{0}=0$ e $\beta_{1}=1$ ). No entanto, a Tabela 4 mostra que a forte correlação dos erros de previsão continua sendo uma característica tanto da amostra completa quanto do período mais recente. Novamente encontram-se evidências que parecem ir contra a hipótese de racionalidade das expectativas.

Tabela 4: Características dos Erros de Previsão - Instituições Top 5

\begin{tabular}{lcccc}
\hline \multicolumn{4}{l}{ correlação dos erros de previsão com $x_{t}$ na defasagem $s$} \\
\hline \multicolumn{4}{l}{ desde janeiro de 2002 (100 observações) } \\
\hline$x_{t}$ & $s=1$ & $s=2$ & $s=3$ & $\ell(3)$ \\
\cline { 2 - 5 }$\pi_{t}-E\left[\pi_{t} \mid I_{t-1}\right]$ & 0,526 & 0,234 & 0,210 & $29,32^{* * *}$ \\
$\Delta \pi_{t}$ & 0,288 & 0,072 & 0,056 & \\
\hline desde março de 2006 (50 últimas observações) & & \\
\hline$x_{t}$ & $s=1$ & $s=2$ & $s=3$ & $\ell(3)$ \\
\cline { 2 - 5 }$\pi_{t}-E\left[\pi_{t} \mid I_{t-1}\right]$ & 0,423 & 0,101 & 0,086 & $11,04^{* *}$ \\
$\Delta \pi_{t}$ & 0,226 & 0,026 & 0,028 & \\
\hline
\end{tabular}

Nota: A estatística $\ell(3)$ é o resultado do teste em que a hipótese nula sustenta que a primeira até a terceira auto-correlação dos erros de previsão é zero.

*** e ** indicam significância estatística no nível de confiança de $1 \%$ e $5 \%$, respectivamente.

\section{HISTERESE DAS EXPECTATIVAS}

Uma vez que a seção anterior sugere a presença de erros sistemáticos nas projeções, cabe investigar o padrão das revisões dessas em maior detalhe. A Figura 2 mostra as distribuições de freqüência tanto da variação da inflação como da variação da projeção mediana do IPCA. As distribuições de frequiência evidenciam o fato de que as variações da projeção têm magnitude consideravelmente menor que as variações da taxa de inflação observada: em $75 \%$ das observações, a variação absoluta da projeção é inferior a 15 pontos-base, enquanto que a variação da inflação mensal fica abaixo deste limiar em somente $43 \%$ dos casos. Enquanto a aceleração absoluta da inflação tem uma média de 22,6 pontos base (e variância de 5,5 p.b.), a variação absoluta média da projeção é de apenas 11,6 pontos-base (variância de 1,4 p.b.).

A histerese das expectativas é confirmada também pelo fato de que a variação absoluta das expectativas excedeu a variação absoluta da inflação em apenas $28 \%$ das observações.

Ressalte-se que este padrão de sub-estimação das variações não é de forma alguma peculiar ao caso brasileiro, sendo também encontrado, por exemplo, no Survey of Professional Forecasters nos Estados Unidos (vide trabalhos de Nordhaus (1987), Evans e Wachtel (1993) e Henzel (2008)). 
Figura 2: Distribuições de Frequência

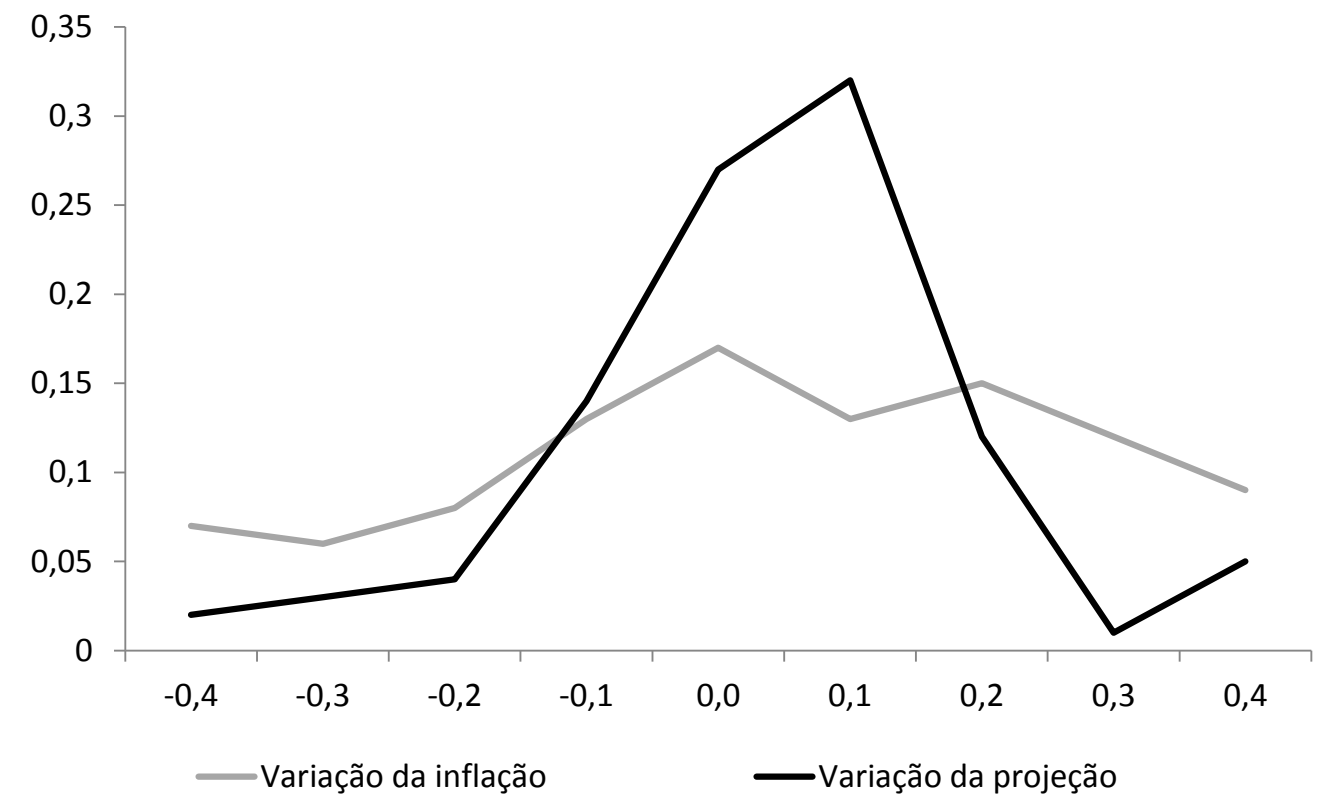




\section{PREVISÕES AJUSTADAS}

A observação de que, como mostramos na Seção 2, erros de previsão estão fortemente correlacionados com erros subseqüentes abre a possibilidade de melhorias no desempenho preditivo. Em outras palavras, podemos tirar proveito do fato de que os erros dos agentes econômicos são previsíveis e persistentes, ajustando as previsões relatadas no Focus de modo a levar em consideração erros de previsão passados. A tabela 5 mostra que tanto o erro absoluto médio (MAE) quanto a raiz do erro médio quadrático (RMSE) são reduzidos quando - a título de exemplo - utilizamos a regra de previsão

$$
E_{\text {ajustada }}\left[\pi_{t+1} \mid I_{t}\right]=1,11 \cdot E_{\text {med }}\left[\pi_{t+1} \mid I_{t}\right]+0,55 \cdot\left(\pi_{t}-E_{\text {med }}\left[\pi_{t} \mid I_{t-1}\right]\right)-0,02
$$

Os coeficientes para este ajuste das previsões foram obtidos pela regressão da inflação ocorrida contra os regressores do lado direito da expressão acima, utilizando-nos de dados até dezembro de 2007, sem re-estimação posterior. A estatística $t$ para o termo "erro de previsão no mês anterior" foi de 4,77 , sugerindo que tal erro é de fato altamente significativo para a previsão de inflação. ${ }^{4}$

Tabela 5: Erro Absoluto Médio e Raiz do EMQ das Previsões de Inflação

\begin{tabular}{ccc}
\hline projeção & MAE & RMSE \\
\hline univariada com AR(1) & 0,1921 & 0,2207
\end{tabular}

previsões de dezembro de 2007

\begin{tabular}{ccc}
$\begin{array}{c}\text { mediana do FOCUS } \\
\text { previsão ajustada }\end{array}$ & 0,1311 & 0,1631 \\
\hline Nota: Estatísticas computadas para o & 0,1484 \\
\hline
\end{tabular}

\section{CONCLUSÕES}

Do que foi exposto acima, conclui-se que um modelo de formação de expectativas que vise explicar as projeções de inflação no Brasil deve necessariamente ser capaz de explicar os seguintes fatos estilizados:

i) o padrão de auto-correlação nos erros de previsão;

ii) a correlação entre aumentos na taxa de inflação e sub-estimações subsequentes da inflação, e,

iii) a histerese nas projeções.

Em função disto, os modelos em que observações passadas da inflação têm maior peso na composição de expectativas médias correntes (como o modelo de expectativas adaptativas ou o modelo de rigidez de informação), parecem mais adequados do que a suposição de expectativas racionais.

À primeira vista os resultados encontrados aqui parecem estar relativamente alinhados com os encontrados por Guillén (2008) - que também rejeita a hipótese de expectativas racionais. No entanto, a rejeição da hipótese de expectativas racionais para o grupo das instituições Top 5 em algumas amostras sugere cuidado na aplicação direta do modelo de rigidez de informação de Mankiw e Reis ao contexto

\footnotetext{
${ }^{4} \mathrm{O} R 2$ ajustado da regressão foi de 0,677 , contra 0,567 quando o erro de previsão do período anterior foi ignorado.
} 
brasileiro. Mesmo que esse modelo seja naturalmente atrativo pela sua capacidade de explicar porque o impacto máximo de mudanças na política monetária ocorre com defasagem de vários meses.

A validação do modelo de rigidez de informação deve necessariamente estar condicionada à validação da hipótese de que ao menos os agentes que revisam suas expectativas o fazem de forma racional. Não nos foi possível obter tal confirmação aqui. Além disto, a suposição de que uma instituição leva vários meses para atualizar suas informações também nos parece inadequada para o conjunto de profissionais que participam da pesquisa do Banco Central, já que estes tendem a acompanhar os indicadores macroeconômicos continuamente. O modelo de rigidez de informação freqüentemente é apresentado como alternativa mais simples aos modelos de bounded rationality. As considerações acima sugerem que infelizmente a simplicidade pode estar vindo acompanhada da imprecisão.

\section{BIBLIOGRAFIA}

Brown, R., Durbin, J., \& Evans, J. (1975). Techniques for testing the constancy of regression relationships over time. Journal of the Royal Statistical Society, 37(2):149-163.

Cumby, R. \& Huizinga, J. (1992). Testing the autocorrelation structure of disturbances in ordinary least squares and instrumental variables regressions. Econometrica, 60(1):185-198.

Evans, M. \& Wachtel, P. (1993). Inflation regimes and the sources of inflation uncertainty. Journal of Money, Credit and Banking, 25(3):475-511.

Guillén, D. (2008). Expectativas de inflação no Brasil: Racionais, adaptativas ou sticky information. Seminário DIMAC 295, Ipea.

Henzel, S. (2008). Learning trend inflation - Can signal extraction explain survey forecasts? Munich, Ifo - Institute for Economic Research.

Kohlscheen, E. (2010). Uma nota sobre erros de previsão da inflação de curto-prazo. Banco Central do Brasil. Working paper, 227.

Mankiw, N. \& Reis, R. (2002). Sticky information vs. sticky prices: A proposal to replace the new keynesian phillips curve. Quarterly Journal of Economics, 117(4):1295-1328.

Mankiw, N., Reis, R., \& Wolfers, J. (2004). Disagreement about inflation expectations. Working Paper 9796, NBER.

Newey, W. \& West, K. (1987). A simple, positive semi-definite, heteroskedasticity and autocorrelation consistent covariance matrix. Econometrica, 55(3):703-708.

Nordhaus, W. D. (1987). Forecast efficiency: Concepts and applications. Review of Economics and Statistics, 69(4):667-674. 